\title{
Interface Assignment-Based AODV Routing Protocol to Improve Reliability in Multi-Interface Multichannel Wireless Mesh Networks
}

\author{
Won-Suk Kim and Sang-Hwa Chung \\ Department of Computer Engineering, Pusan National University, Busan 609-735, Republic of Korea \\ Correspondence should be addressed to Sang-Hwa Chung; shchung@pusan.ac.kr
}

Received 4 August 2013; Accepted 22 October 2013

Academic Editor: David Taniar

Copyright (C) 2015 W.-S. Kim and S.-H. Chung. This is an open access article distributed under the Creative Commons Attribution License, which permits unrestricted use, distribution, and reproduction in any medium, provided the original work is properly cited.

\begin{abstract}
The utilization of wireless mesh networks (WMNs) has greatly increased, and the multi-interface multichannel (MIMC) technic has been widely used for the backbone network. Unfortunately, the ad hoc on-demand distance vector (AODV) routing protocol defined in the IEEE 802.11s standard was designed for WMNs using the single-interface single-channel technic. So, we define a problem that happens when the legacy AODV is used in MIMC WMNs and propose an interface assignment-based AODV (IAAODV) in order to resolve that problem. IA-AODV, which is based on multitarget path request, consists of the PREQ prediction scheme, the PREQ loss recovery scheme, and the PREQ sender assignment scheme. A detailed operation according to various network conditions and services is introduced, and the routing efficiency and network reliability of a network using IA-AODV are analyzed over the presented system model. Finally, after a real-world test-bed for MIMC WMNs using the IA-AODV routing protocol is implemented, the various indicators of the network are evaluated through experiments. When the proposed routing protocol is compared with the existing AODV routing protocol, it performs the path update using only $14.33 \%$ of the management frames, completely removes the routing malfunction, and reduces the UDP packet loss ratio by $0.0012 \%$.
\end{abstract}

\section{Introduction}

Wireless Mesh Networks and Related Technics. Wireless mesh networks (WMNs) are regarded as a next-generation technology because they can provide high network extensibility and are economical. WMNs research based on IEEE 802.11s, which is an IEEE 802.11 amendment for mesh networking, is actively progressing [1]. WMNs have been selected as backbone networks in many places due to ability to provide network stability and reliability of data transfer in wireless sensor networks, smart city applications, and so forth.

One of the ways to improve network efficiency is the multi-interface multichannel (MIMC) technic, which uses multiple wireless channels via multiple interfaces. The MIMC technic minimizes interference on the same channel and prevents degradation of throughput, even though the number of hops increases [2-5]. In addition, the quality of each link in the network has improved through the use of directional antennas $[6,7]$.
The ad hoc on-demand distance vector (AODV) routing protocol defined in the IEEE 802.11s standard is widely employed in ad hoc networks and WMNs. The basic operations of AODV using the on-demand routing scheme are as follows. First, a source node that wants to create a new path broadcasts a path request (PREQ) frame including information on the target node. All nodes receiving that management (MGMT) frame generate a backward path toward the source node. If the receiving node is the target of the PREQ, it will send a path reply (PREP) frame to the source node via unicast; otherwise, the PREQ is simply rebroadcast. The PREP sent by the target node is delivered to the source node, and the mesh path is created when the source node receives the PREP. In addition, the source node performs a path update by sending a PREQ periodically in order to find a path with a better metric [8].

Research Motivation and Contribution. As mentioned earlier, WMNs have been utilized as backbone networks in various 
fields. To enhance the valuation of WMNs, not only must capacity improve, but also efficiency and reliability must be increased. One of the best ways to enhance WMNs is to take advantage of the MIMC technic and the directional antenna. In WMNs that adopt the MIMC technic and directional antenna (MIMC WMNs), additional technical requirements for the routing protocol improve efficiency and reliability.

Actual traffic flows should also be considered, but most routing protocols take into consideration only traffic between the mesh portal and the mesh routers. If network scale expands or a specific application that generates a lot of internal network traffic is in service, traffic flow may not be directed to the mesh portal $[9,10]$. In this case, the overhead of the path update process will greatly increase, so it must be handled by the routing protocol.

In this paper, we define the problems that occur when an existing AODV is used in MIMC WMNs, and we present a new routing protocol that improves routing efficiency and network reliability. The existing issues and the research contributions for MIMC WMNs are as follows.

(i) In the original AODV using the single-target PREQ (ST-PREQ), a structural problem exists where the PREQ frame cannot be delivered through the normal path. Because of this, the mesh path will change unintentionally. However, the proposed interface assignment-based AODV (IA-AODV) can resolve this problem by using a multitarget PREQ (MTPREQ), which is the MGMT frame performing a path update with multiple targets at the same time.

(ii) When a PREQ is received through multiple interfaces, the random receiving order of the PREQ shows a low correlation between the routing metric and the PREQ receiving order. Because of this problem, frequent replacement of the interface responsible for next-hop communication occurs, and network reliability will be degraded from the decrease in packet delivery ratio and an increase in the routing malfunction ratio. The PREQ random receiving order problem is resolved by a PREQ prediction scheme in IA-AODV.

(iii) If the network scale is enlarged and internal network paths increase, the efficiency of routing will decline due to increasing of the MGMT frames for the routing update process. On the other hand, IA-AODV is able to maximize routing efficiency by using MT-PREQ and a PREQ prediction scheme, handling exceptional situations such as PREQ loss.

(iv) The simple rule of the PREQ sender decision reduces efficiency of the routing protocol, but IA-AODV improves routing efficiency by using a PREQ sender assignment scheme.

Organization. The remainder of this paper is organized as follows. Section 2 presents background and related works, and Section 3 presents the system model for MIMC WMNs and defines the problem. In Section 4, IA-AODV is explained in detail, and in Section 5 the experimental results are analyzed. Finally, we conclude this paper in Section 6.

\section{Background and Related Works}

2.1. Background of the AODV Routing Protocol. The MIMC WMNs presented in this paper use a directional antenna, so the link quality of most mesh links within MIMC WMNs is guaranteed to be high; therefore, the result of the update process to find a better path will be diminished. So, when the legacy AODV routing protocol is employed in MIMC WMNs, there are many points to consider, unlike singleinterface single-channel (SISC) WMNs. First, the routing update process is an essential factor to find the best path during communications, but if the wireless channel information is not frequently changed then the number of MGMT frame transmissions in the update process will be an overhead. Therefore, a scheme that minimizes the MGMT frames in every update period is required. Second, routing malfunctions occur frequently due to the random receiving order of the PREQs and the PREQ loss, so additional technics are required to solve this problem.

The MT-PREQ, which efficiently reduces the number of PREQ frames, was presented in IEEE 802.11s. If WMNs take advantage of MT-PREQ, then the objective of path discovery or path update with multiple nodes will be achieved with a single PREQ. In IEEE 802.11s, the fields for the MT-PREQ are Target Count, which indicates the number of target nodes, and Per Target, which includes information on each target, such as 1 byte for flag, 6 bytes for medium access control (MAC) address, and 4 bytes for sequence number. (The Per Target field is referred to as the target list in this paper.) The main technics of the IA-AODV proposed in this paper are based on MT-PREQ.

2.2. Related Works. Currently, a lot of research into routing schemes or broadcast schemes for ad hoc networks and WMNs has been in progress. The main goals of these works are interference avoidance within the network, improvement in packet delivery ratio, and increasing end-to-end bandwidth. Other researches focus on MIMC WMNs with a directional antenna for high-performance backbone networks.

From our own previous work on MIMC WMNs, the channel load aware routing protocol, the multipath AODV protocol for fast recovery, and implementation of IEEE $802.11 \mathrm{n}$ multihop have been studied to increase of link bandwidth [11-13]. The channel load aware routing protocol selects the most efficient multihop path via channel usage in multiple paths between the source node and the target node [11], and the multipath AODV protocol searches for an alternative path when the original path is compromised [12]. In this paper which extends earlier research [14], an efficient and novel routing protocol focuses on maximizing routing efficiency and network reliability while minimizing overhead in the routing process.

Several studies of routing protocols were carried out to improve the performance of mobile ad hoc networks (MANETs) and WMNs [15-20]. There is implementation of AODV in the earliest forms of WMNs $[18,20]$, and a routing protocol using a cache to increase the packet delivery ratio in an ad hoc network with high mobility was proposed [17]. In addition, the hybrid wireless mesh protocol (HWMP) was 
studied for communication with the mesh portal as well as internal communication in WMNs [19]. The node type aware AODV routing protocol in hybrid WMNs, which includes not only a mesh router but also clients, was proposed [15]. On the other hand, the proposed routing protocol achieves improvement of network throughput by maximizing routing efficiency and network reliability.

A vast amount of research concentrates on routing protocols for MIMC WMNs to improve performance through avoidance of interference $[4,5,21-25]$. T. C. Tsai and S. T. Tsai [25] proposed a cross-layer routing protocol that controls the transmit power of each network interface in MIMC WMNs to increase performance. Paschoalino and Madeira [23] demonstrated a scalability and link quality aware shortest path routing protocol in order to improve throughput. In addition, Subramaniam et al. [21] and Liu and Liao [24] presented a routing protocol using signal-to-interferenceplus-noise ratio (SINR) as interference value and expected transmission time (ETT) in MIMC WMNs. In contrast, IAAODV modifies the existing AODV to improve network reliability and supports scalability without management overhead.

Anker et al. [26] proposed an AODV routing protocol to improve network reliability by preventing a selfish node from concentrating only on power savings without data delivery in a MANET. As a result, it achieved quality of service (QoS) guarantees and an improved packet delivery ratio and was then verified through simulation of the amount of remaining packets in the buffer and the routing malfunction rate. Boice et al. [27] demonstrated a routing protocol that efficiently ensures buffer operation for intermittently connected networks. This routing protocol operates according to the current status of the connection and the buffer. In this way, it increases packet delivery ratio and reliability, but it also reduces the number of routing messages. Unlike other researches [26, 27], a specialized routing protocol for MIMC WMNs with directional antennas as a high-performance wireless backbone network is proposed and was evaluated in terms of reliability of the network and performance increase through actual implementation.

As described above, research into routing protocols to improve performance of MIMC WMNs has been plentiful. However, most of these studies assume ideal conditions and do not consider practical issues. IA-AODV considers features of MIMC WMNs employed in backbone networks in various fields and achieves network reliability through a propagation method for routing MGMT frames based on interface assignment. In addition, it also achieves routing efficiency by decreasing routing overhead based on designation by the management frames' sender.

\section{System Model and Problem Statement}

3.1. System Model of MIMC WMNs. A mesh node in MIMC WMNs can have multiple interfaces that conduct communication through different channels. Also, because it uses a directional antenna, each interface is responsible for connection with only one neighbor node. A mesh link is created

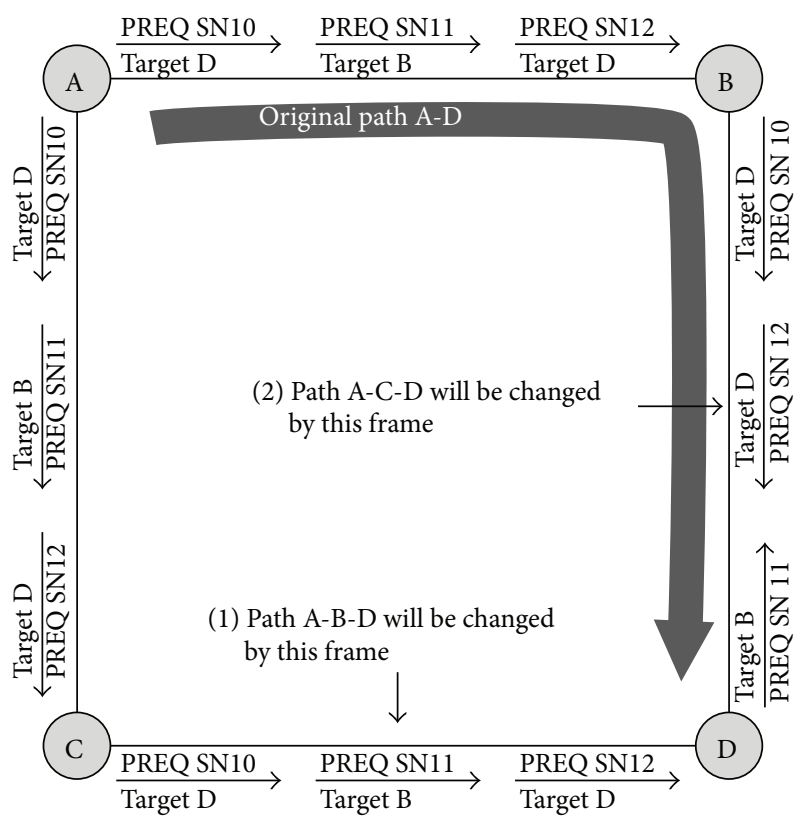

FIGURE 1: The structural problem of AODV using ST-PREQ.

between two interfaces, so the multihop link consists of the number of interfaces, which is the same as double the number of hops. In terms of path changes through information update in the routing mechanism, considerable differences exist in MIMC and SISC. With SISC, change of the next-hop through a path change generates overhead to modify only the receive address (RA) of the MAC header of each frame in the transmit queue. On the other hand, modification of the nexthop in MIMC adds overhead that not only changes the RA of each frame but also resets the appropriate interface for the RA. A single mesh engine controls and manages the multiple interfaces. So, the overhead of above operation that requires a locking mechanism is greatly increased. In addition, the broadcast frame in MIMC WMNs is copied as the number of interfaces in the mesh node and is then sent over each interface separately.

MIMC WMNs employed as a backbone are based on a wireless link, so link quality will absolutely affect the value of the network. Therefore, most industrial MIMC WMNs use a directional antenna. When a directional antenna is used, collision and interference on the same channel are reduced and the reach of the signal is increased, so it can significantly improve link quality. In addition, the logical topology becomes equal to the physical topology.

\subsection{Problem Statement}

3.2.1. Structural Problem of AODV Using ST-PREQ. If an existing AODV using ST-PREQ is adopted in MIMC WMNs, the structural problem of routing will occur, as shown in Figure 1. In Figure 1, the active paths are indicated as A-B and $\mathrm{A}-\mathrm{D}$; in particular, the path between nodes $\mathrm{A}$ and $\mathrm{D}$ is formed by A-B-D, which has a better metric. In order to update the path information, node A sends two PREQs every 


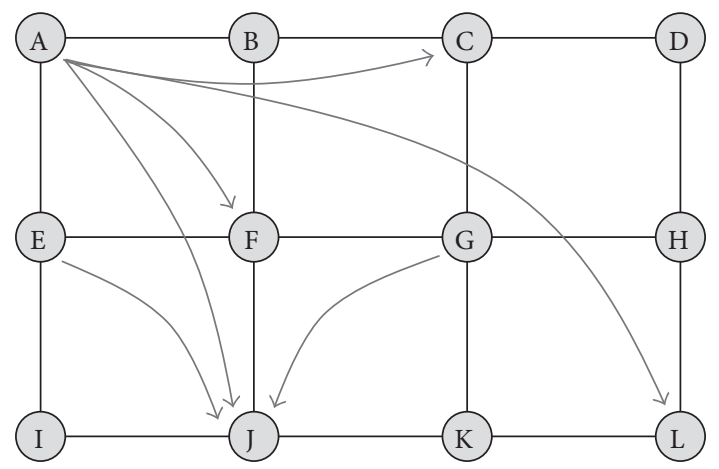

FIgURE 2: An example of MIMC WMN topology and traffic flow.

update period, and each PREQ includes target nodes B and D, respectively. Every PREQ will be sent through all interfaces of node in order to update mesh paths. The PREQ, which has sequence number (SN) 10 , is transferred to node $\mathrm{D}$ as the target. It will pass through nodes $\mathrm{B}$ and $\mathrm{C}$ and will arrive at node D. The PREQ with SN 11 is broadcast to node B as the target; one of the copies of the PREQ is directly delivered to node $B$ and another reaches node $B$ via nodes $C$ and $D$. At this time, node $D$ receives the PREQ, which has the latest $\mathrm{SN}$ of node $\mathrm{A}$ from only node $\mathrm{C}$. Therefore, node $\mathrm{D}$ sets the nexthop towards node $\mathrm{A}$ to node $\mathrm{C}$, because it misunderstands that the path A-B-D is not valid anymore. After that, node A sends the PREQ with SN 12 to node D as the target through nodes $B$ and $C$; the path $A-D$ will be replaced with $A-B-D$ with a better metric. This structural problem from ST-PREQ may occur more frequently in a topology including a multipath. Thus, when the network scale is larger, this problem will occur multiple times during one update period. In addition, this problem may become a serious situation when one node takes charge of sending PREQ/PREP at the same time.

\subsubsection{Increasing Routing Complexity Problem of AODV Using} $S T$-PREQ. Figure 2 shows an example of PREQ transmissions to update and maintain paths in small-scale MIMC WMNs. When the existing AODV routing protocol is applied, the number of PREQ frames required within one path update period is expressed in

$$
N_{\text {PREQ }}=\sum_{i}^{N_{\text {src }}} N_{\text {target }(i)} .
$$

In (1), $N_{\text {src }}$ is the number of mesh nodes responsible for transmitting the PREQ in MIMC WMNs. $N_{\text {target( }(i)}$ means the number of target nodes that communicate with the $i$ th source node. For example, in the topology of Figure 2, there are 6 paths (A-C, A-F, A-J, A-L, E-J, and G-J). In that case, the mesh nodes responsible for transmission of the PREQ are nodes A, E, and G. At this time, $N_{\text {src }}$ is $3 ; N_{\text {target(A,E,G) }}$ is 4,1 , and 1. Therefore, the number of total PREQ frames within the routing update period is $4+1+1=6$. It is equal to the number of paths.

In a general broadcast mechanism (flooding-if the broadcast packet is received, it will just be rebroadcast), the total number of transmissions in the propagation process of the $i$ th PREQ to the whole network is defined in

$$
N_{\text {broadcast }(i)}=\sum_{j=1}^{n} N_{\operatorname{IF}\left(X_{j}\right)}-N_{\operatorname{IF}(\operatorname{target}(i))} .
$$

In (2), $N_{\mathrm{IF}\left(X_{j}\right)}$ indicates the interface number of the $j$ th mesh node, and $N_{\mathrm{IF}(\operatorname{target}(i))}$ reflects the interface number of the target node that receives the ith PREQ. Each mesh node in MIMC WMNs has the interface as the number of links; therefore, the sum of interfaces of all mesh nodes is twice the number of all mesh links. In Figure 2, the total number of broadcasts in the path update period of the path $\mathrm{A}-\mathrm{C}$ is $34-3=31$. Consider

$$
N_{\text {total } t x}=\sum_{i}^{N_{\text {PREQ }}} N_{\text {broadcast }(i)} .
$$

Equation (3) presents the total number of transfers for PREQ propagation within the path update period. Using the example in Figure 2, $N_{\text {total tx }}$ is $31+30+31+32+$ $31+31=186$. Along with the structural problems from ST-PREQ, the total number of transfers of MGMT frames will greatly increase when the network scale becomes larger or the internal network paths increase. In particular, each mesh node in the MIMC WMN has the interface as the number of links; therefore, copies of the broadcast frame as the number of interfaces are transmitted. As a result, delay in operation and transmission, waste of network resources, and complexity of the network management process increase significantly.

3.2.3. The Random Receiving Order of PREQ and the PREQ Loss Problem. In addition to the above problems, the random receiving order of PREQ problem exists. When a PREQ is received through multiple interfaces, the random receiving order of PREQ problem has a low correlation between the routing metric and PREQ receiving order. In other words, when the path update is performed, the next-hop towards the PREQ source node changes according to the first receipt of the latest PREQ via the poor path; then the next-hop towards the PREQ source changes again with the next receipt of the same PREQ via the good path. This problem will occur more frequently if there are more PREQ transmissions and multipaths.

Routing failure caused by PREQ loss also exists. The PREQ is propagated as broadcast by default; it is transmitted after physical carrier sensing is conducted in the MAC layer. Also, the PREQ frame does not require acknowledgement (ACK). Because of these factors, the loss ratio of the PREQ appears quite high. Commonly, PREQ loss is recovered in the next path update, but loss in a link near the source node makes the routing failure affect the entire network. Therefore, PREQ loss may degrade network reliability. The relation of routing error rate and loss rate per link is highly dependent on the location where the link loss occurs. 


\section{Interface Assignment-Based AODV (IA-AODV) Routing Protocol}

IA-AODV is based on MT-PREQ in IEEE 802.11s. The basic operation for MT-PREQ is as follows. When the node receives the PREQ, it checks the target list. If the target list contains the address of the node receiving the PREQ, the node will send a PREP to the source node and remove its own address from the target list. After that, if any addresses remain in the target list, then the PREQ is rebroadcast. Also, the node that has the responsibility for transmission of the PREQ can conduct a path update for all targets with transmission of a single MTPREQ. With this mechanism, $N_{\text {target }(i)}$ in (1) is replaced with 1 , so (1) can be simplified to

$$
N_{\text {PREQ }}=N_{\text {src }} \text {. }
$$

It means that the node that has responsibility for transmission of the PREQ can transmit only one MT-PREQ rather than the number of targets. Therefore, the PREQ frames up to the number of the PREQ source nodes are propagated during one path update period.

\subsection{PREQ Prediction Scheme}

4.1.1. Basic Operation of PREQ Prediction. In an existing AODV using MT-PREQ, the use of a simple method of operation as mentioned above is inefficient in MIMC WMNs. In MIMC WMNs, only one neighbor is connected by one interface, so there is no need to transmit the PREQ via an interface that already received another PREQ that has a better metric from the same source node. In addition, it is possible to transmit a PREQ that contains integrated information of received PREQs from other interfaces, and it is also possible to send frames that include predicted information of the PREQ and which are received periodically. The proposed PREQ prediction scheme for MT-PREQ, along with the above concepts, has the following features.

(i) When the interface is initially assigned, the role of the interface is assigned as receiving or transmitting the PREQ, and then the number of PREQ frames can be reduced efficiently through this role. The interface assigned the role of PREQ receiving is called an IN interface; otherwise, it is an OUT interface.

(ii) All OUT interfaces are configured to send the required target list which is combined with information of the target list from each IN interface.

(iii) In the path update process, the predictive PREQ will be transmitted via all OUT interfaces, even if the PREQ is not received through all IN interfaces. In this way, the PREQ is rapidly propagated, and the PREQ random receiving order problem can be resolved.

(iv) With the IN interface, due to the routing, protocol can immediately handle the change of routing information or the PREQ loss, and improvement of routing efficiency and network reliability can be achieved.
TABLE 1: Example of a PREQ information table.

\begin{tabular}{lccccc}
\hline Interface & Direction & Targets & SN & Metric & Exp_time \\
\hline 0 & IN & F, G, L & 11 & 468 & $300 \mathrm{~ms}$ \\
1 & OUT & F, L & 11 & 612 & $301 \mathrm{~ms}$ \\
2 & OUT & F, L & 11 & 612 & $301 \mathrm{~ms}$ \\
3 & IN & E, F, L & 11 & 407 & $320 \mathrm{~ms}$ \\
\hline
\end{tabular}

Figure 3 represents the flow of PREQ propagation in MIMC WMNs. Figures 3(a) and 3(c) show PREQ propagation when the PREQ prediction scheme is not used, and Figures 3(b) and 3(d) indicate the PREQ propagation with the PREQ prediction scheme. In Figure 3(a), node A broadcasts the PREQ, which has node $\mathrm{L}$ as a target; all forwarding nodes receiving that frame rebroadcast it using all their own interfaces. As shown in Figure 3(b), the PREQ is propagated in an efficient way by the role assignment of interfaces. This is possible because the role of interfaces is preallocated by classifying the PREQ that has a good metric and the other PREQ.

The process of path update with multiple targets is represented in Figure 3(c). The A- $(\mathrm{E}) / \mathrm{F} /(\mathrm{G}) / \mathrm{L}$ means that the inclusion of nodes $\mathrm{E}$ and $\mathrm{G}$ was not determined in the corresponding PREQ, if the PREQ is received via a path that has a good metric relatively late; then the other PREQ, which has the same SN and a different target list, will be transmitted earlier. This issue will occur more frequently at the node far from the PREQ source node, and it brings on degradation of network reliability by increasing routing complexity. However, Figure 3(d) presents the PREQ prediction scheme that affects the path update with multiple targets; thus, all PREQs are propagated through the whole network through the most suitable path.

The PREQ prediction scheme adopted to propagate MTPREQ more efficiently on MIMC WMNs can reduce the number of transmissions during the path update period by removal of unnecessary and duplicated broadcasts in each link. As a result, this scheme contributes to (2), which means the total number of transmissions to propagate the $i$ th PREQ can be replaced with (5). Consider

$$
\begin{gathered}
N_{\text {broadcast }}=\frac{\sum_{i=1}^{n} N_{\mathrm{IF}\left(X_{i}\right)},}{2} \\
N_{\text {total } t x}=N_{\text {PREQ }} \times N_{\text {broadcast }},
\end{gathered}
$$

where $N_{\text {broadcast }}$ is the same as the number of all links in the network; it can be an independent value from the source or target node of the PREQ. Thus (3) can be simplified to (6), and the $N_{\text {total } t x}$ in Figure 2 is $3 \times 17=51$.

4.1.2. PREQ Information Table. For the PREQ prediction scheme, classification by the source node of the information of the received PREQ is required. The PREQ information table (PIT) is utilized for the classification, and it will exist in all mesh nodes. The number of PITs in each node is the same as the number of the PREQ source nodes. In a specific mesh node, the PIT for node A, which is the source node of the PREQ, is shown in Table 1. The PIT has data entries as the 


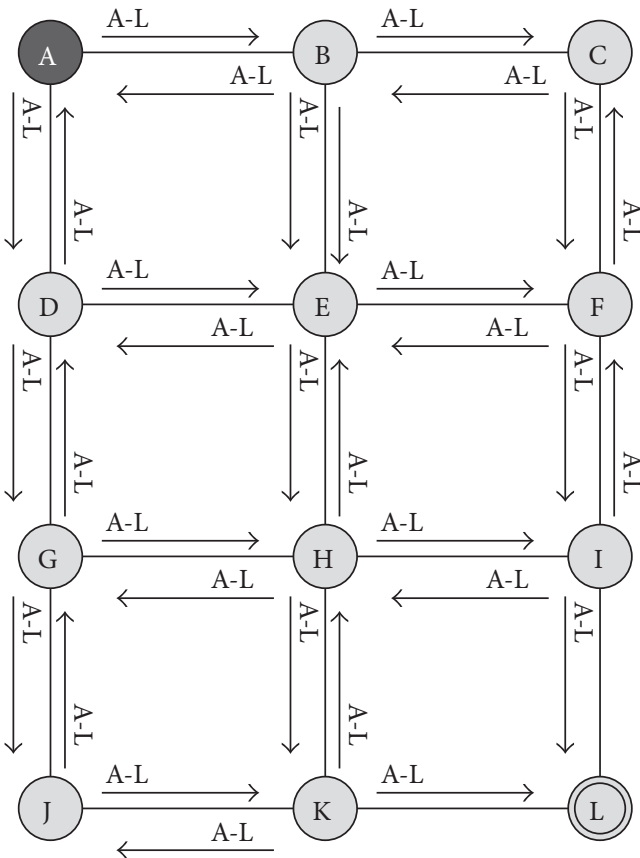

(a) Single target without PREQ prediction scheme
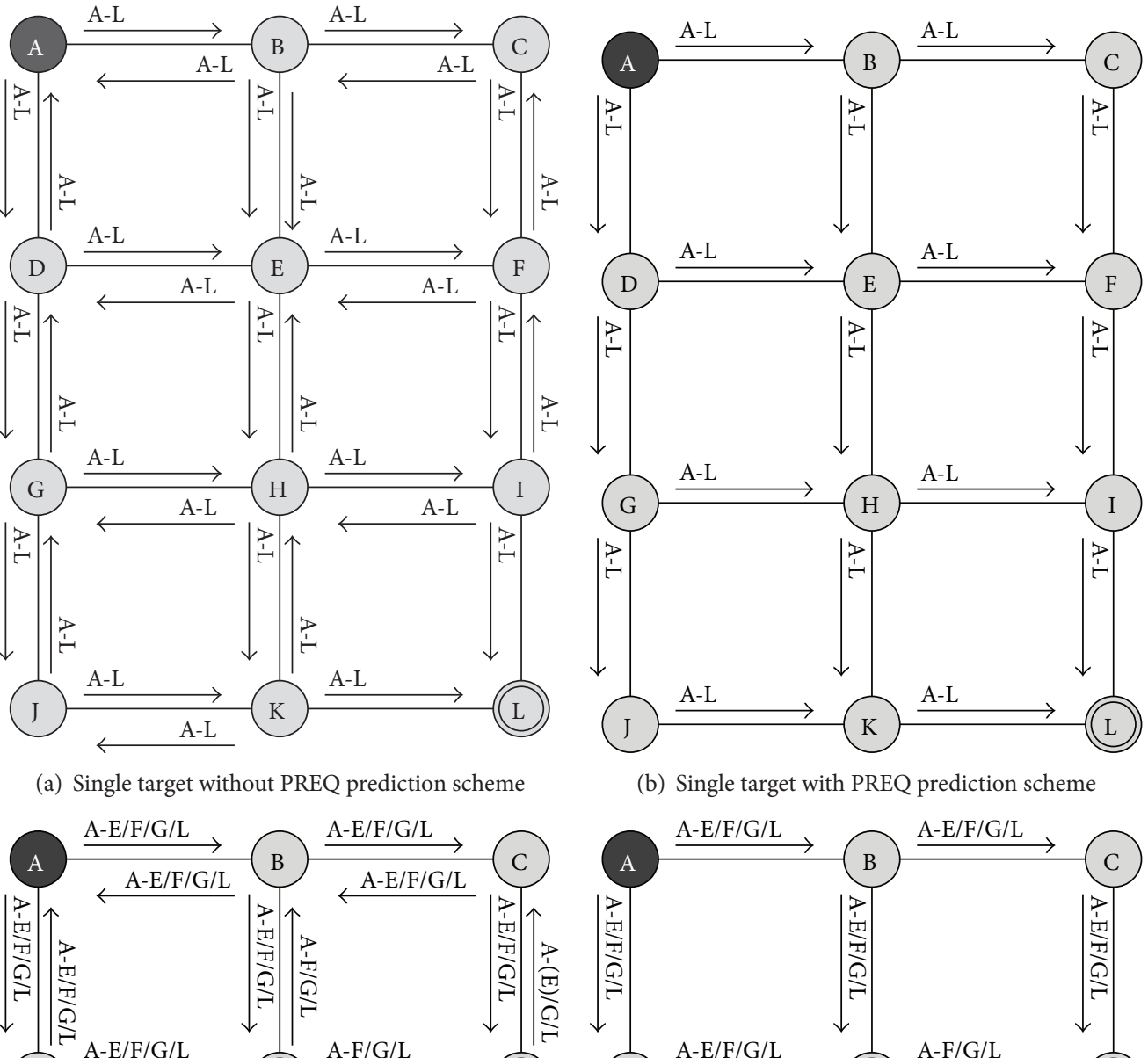

(b) Single target with PREQ prediction scheme
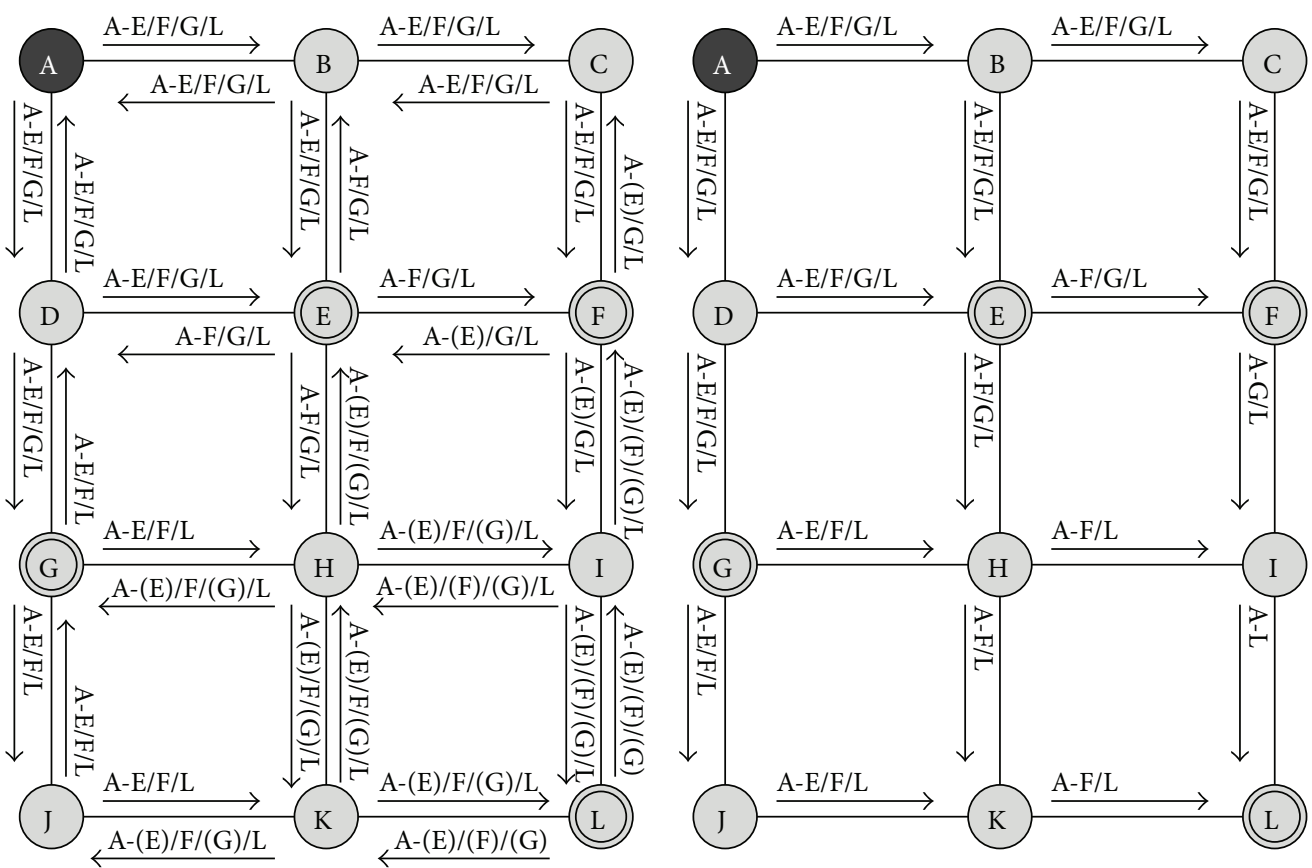

$\begin{array}{ll}\text { A Source node } & \text { (E) Forwarding node } \\ \text { (F) Target node } & \text { A-C/F }\end{array}$

(c) Multiple target without PREQ prediction scheme

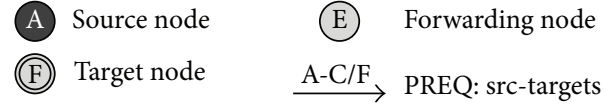

(d) Multiple target with PREQ prediction scheme

FIgure 3: Applying the PREQ prediction scheme. 


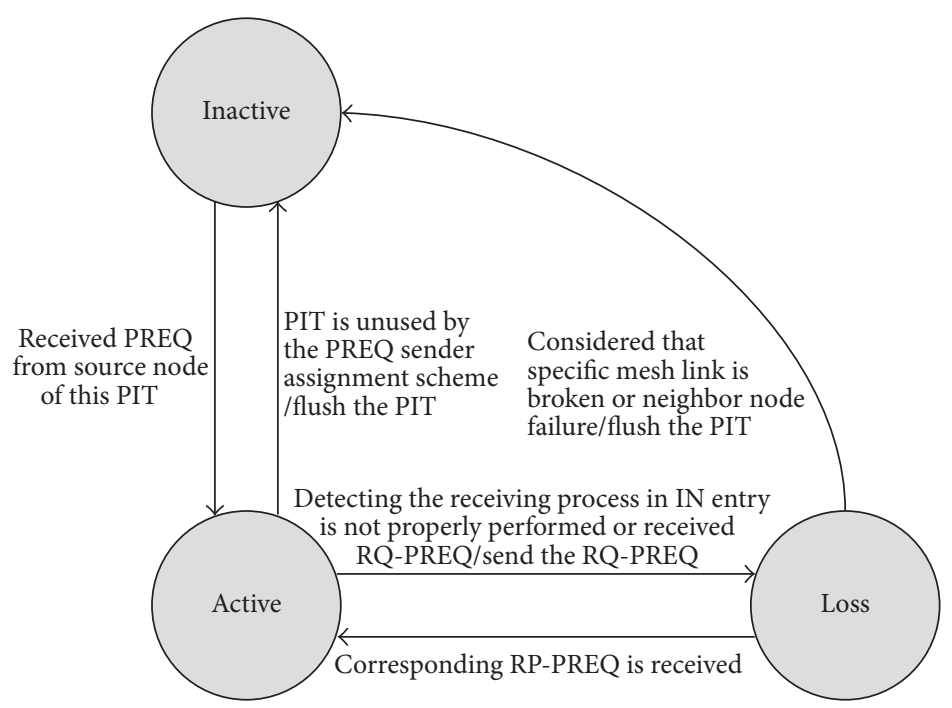

FIGURE 4: The state transition diagram of the PIT state.

number of the interfaces of the node, and the meaning of each field is as follows.

(i) Interface. The index of the interface within the mesh node.

(ii) Direction. The role assigned to the interface; it can have one of three values: NULL, IN (receiving), and OUT (transmitting).

(iii) Targets. The calculated target list of the recent PREQ.

(iv) SN. The sequence number of the received or transmitted PREQ.

(v) Metric. The routing metric of the PREQ that passed through this interface.

(vi) Exp_time. The elapse time from the last PREQ.

In particular, each entry of the PIT is mapped to a specific interface by the interface field. The direction field of entry sets the role of the corresponding interface.

4.1.3. States for PIT. All PITs within mesh nodes in MIMC WMNs using the PREQ prediction scheme have one of three states, as seen in Figure 4. When the PREQ is received, if the PIT that is relevant to the source node of the PREQ does not exist, the PIT and entry will be set, and the state of the PIT becomes active. In the active state, the initial setup of the PIT and the assigning of the interface are performed, and the PIT is continually updated based on the information of the received PREQ in every path update. If the PREQ is not received through the IN interface within the time limit, the state of the PIT will be changed to a loss state, and then a recovery request PREQ (RQ-PREQ) frame will be sent through the appropriate interface. In the loss state, if the recovery reply PREQ (RP-PREQ) is not received within the time limit, it is possible that the specific mesh link is broken or a neighbor node has failed. So, all entries of the PIT will be flushed, and the state will change to inactive. The behaviors relating to the loss state will be covered in more detail in Section 4.2. In addition, the state of the PIT may change to inactive through the PREQ sender assignment scheme. This will be discussed in detail in Section 4.3.

The PREQ prediction scheme can react immediately against the change in routing information from the PIT state transition. In addition, it can distinguish a temporary PREQ loss and a topology change from a broken link or a node failure and so can greatly improve network reliability.

4.1.4. Processing Details for PREQ Prediction Scheme. The algorithms in the remainder of the paper use the expressions defined in Expression by PREQ Prediction Algorithms. The behaviors of the PIT depend on the state of the PIT. In the active state, which is the initial state, the setting of the PIT related to the source node of the PREQ is completed in all mesh nodes within only one period of PREQ propagation. The algorithm that as PIT in the active state follows is shown in Algorithm 1.

The algorithm in Algorithm 1 is divided into two parts. Lines 1 to 11 form the first part, when the PREQ is received, and lines 12 to 24 form the second part, when the PREQ is transmitted. When the PREQ is received, the PIT related to the source node of the PREQ is searched, and the entry that has same index of the interface as the receiving interface is brought from the PIT. Then, the following operation is performed by comparing the direction of entry. In line 5 , the NULL direction of entry means that no PREQ received or transmitted passed through this interface, so the role of the interface is assigned as Receive. In line 6, the OUT direction indicates that the PREQ received earlier was already sent using this interface; thus, the interface is assigned to IN after comparing the metric. In other words, if this interface can have a better metric when the PREQ is received, then the interface will be an IN interface. Otherwise, it becomes an OUT interface. In line 9, the IN direction shows that the corresponding interface has been receiving already, so if there 
(1) Receive PREQ and search PIT

Active State Algorithm

(2) $e^{\text {in }} \leftarrow$ PIT entry which matched with receiving interface

(3) $p^{\text {in }} \leftarrow$ received $P R E Q$

(4) $\operatorname{switch}\left(e_{d}^{\mathrm{in}}\right)$

(5) case NULL: $e_{t, s, m}^{\text {in }} \leftarrow p_{t, s, m}^{\text {in }}, e_{d}^{\text {in }} \leftarrow I N$

(6) case OUT: $m^{\text {in }} \leftarrow p_{m}^{\text {in }}-m_{\text {last }}$

(7) if $\left(e_{m}^{\text {in }}\right.$ is better than $\left.m^{\text {in }}\right)$ drop PREQ

(8) $\quad$ else $e_{t, s, m}^{\text {in }} \leftarrow p_{t, s, m}^{\text {in }}, e_{d}^{\text {in }} \leftarrow I N$

(9) case IN:

(10) if $\left(e_{t, s, m}^{\text {in }}=p_{t, s, m}^{\text {in }}\right)$ drop PREQ

(11) $\quad$ else $e_{t, s, m}^{\text {in }} \leftarrow p_{t, s, m}^{\text {in }}$

(12) Process AODV routing and prepare transmitting PREQ

(13) for each $e \in P I T$ where $e \neq e^{\text {in }}$ do

(14) $t x\left[e_{i}\right] \leftarrow 1$

(15) switch $\left(e_{d}\right)$

(16) case NULL: $e_{t, s, m} \leftarrow p_{t, s, m}^{\text {out }}, e_{d} \leftarrow$ OUT

(17) case $I N$ :

(18) if $\left(e_{m}\right.$ is worse than $\left.p_{m}^{\text {out }}\right) e_{t, s, m} \leftarrow p_{t, s, m}^{\text {out }}, e_{d} \leftarrow$ OUT

(19) $\quad$ else $t x\left[e_{i}\right] \leftarrow 0$

(20) case OUT: $t_{\text {out }} \leftarrow e_{t} \cap p_{t}^{\text {out }}$

(21) if $\left(e_{s}<p_{s}^{\text {out }}\right.$ or $\left(e_{s}=p_{s}^{\text {out }},\left(e_{m} \neq p_{m}^{\text {out }}\right.\right.$ or $\left.\left.\left.e_{t} \neq t_{\text {out }}\right)\right)\right)$

(22)

(23)

(24) end

$e_{s, m} \leftarrow p_{s, m}^{\text {out }}, e_{t} \leftarrow t_{\text {out }}$

(25) transmit PREQ where $t x[\cdots]=1$

Algorithm 1: The algorithm for receiving and transmitting in the active state PIT.

are no changes to the target list, $\mathrm{SN}$, and metric, then the PREQ will be dropped; otherwise, transmitting PREQ will be prepared after the entry update.

When the operation of entry setting related to PREQ receiving is finished, the other entries should be configured to rebroadcast the PREQ. First, in line 12, the PREQ is prepared after the AODV routing process. At this time, the node's own address is removed from the target list of this PREQ. Unlike the operation in PREQ receiving, all entries except the received entry are examined. In line 16, the NULL direction shows that no PREQ was received or transmitted by this interface, so the interface is appointed as a transmitting interface, and the transmit array is set to 1 . In line 17, the IN entry means that the interface role is already allocated as receiving, in order to receive the PREQ from neighbor nodes; thus, the interface is assigned to OUT after comparing the metric. In line 20, there are two cases for the OUT direction. The first is where the transmitting PREQ has more current information than the entry, and the second is where the PREQ has the same $\mathrm{SN}$ as the entry but has modified information from a neighbor node. For these cases, the entry update will be performed if the PREQ has a greater SN than the entry or if the PREQ has the same SN as the entry but the metric or target list is different.

Figure 5 shows an example of the process for the initial PIT setting of node $\mathrm{H}$ in Figure $3(\mathrm{~d})$. When the PREQ is received from node $\mathrm{G}$, the PIT is established as in the table in the left side of Figure 5, and the PREQ is sent through interfaces 0,2 , and 3 . Then, when a PREQ is received from node $\mathrm{E}$, the received PREQ has a better metric than the saved metric in the first entry of the PIT, so the role of the interface mapped with this entry is assigned to IN. In addition, the targets field of entry is reconfigured, and the PREQ is retransmitted through interfaces 2 and 3.

4.1.5. Computational Complexity for PREQ Prediction Scheme. Clearly, additional computation is required for the PREQ prediction scheme, so we should consider the computational cost and complexity for this scheme. Fortunately, most additional computations involve linear operation, except comparison of the targets. The computational cost of searching PIT for the source of PREQ is $O\left(N_{\text {src }}\right)$, but this operation is independent of other operations. Thus, the computational cost for transmitting PREQ process, which has more operations than receiving process, is $O\left(N_{e}\left(N_{T(e)}+N_{T(p)}\right)\right)$, where $N_{e}$ indicates the number of PIT entries, which is the same as the number of interfaces of a node, $N_{T(e)}$ shows the number of targets of each entry, and $N_{T(p)}$ is the number of targets of transmitting PREQ. The maximum number of targets approaches the total number of nodes in the whole network.

4.2. PREQ Loss Recovery Scheme. The PREQ frame is propagated based on the broadcast, so the probability of loss is significantly higher than data frames. In addition, the loss of the PREQ causes a routing malfunction, and it makes the routing protocol misunderstand the availability of the path in which the loss occurs. To overcome this issue, IA-AODV 

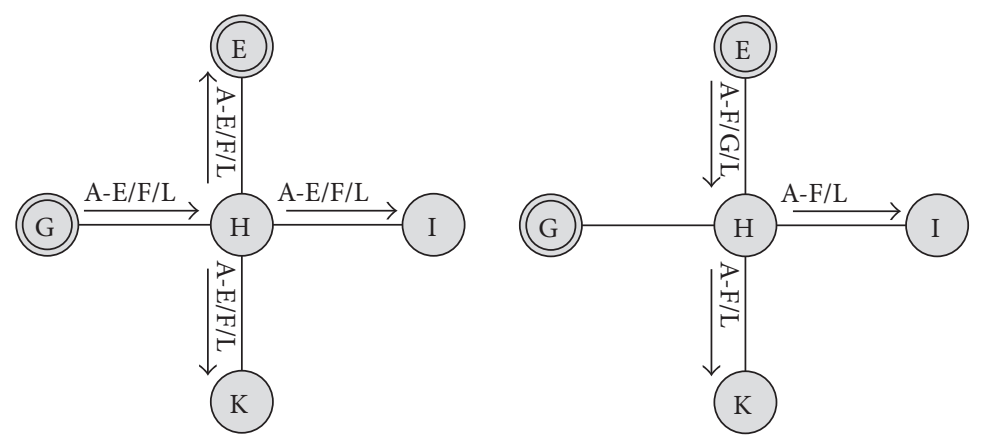

\begin{tabular}{|c|c|c|c|}
\hline Interface & Direction & Target list & Metric \\
\hline $0(\mathrm{E})$ & OUT & E, F, L & 450 \\
\hline $1(\mathrm{G})$ & IN & E, F, L & 320 \\
\hline $2(\mathrm{~K})$ & OUT & E, F, L & 463 \\
\hline $3(\mathrm{I})$ & OUT & E, F, L & 462 \\
\hline
\end{tabular}

\begin{tabular}{|c|c|c|c|}
\hline Interface & Direction & Target list & Metric \\
\hline $0(\mathrm{E})$ & IN & F, G, L & 310 \\
\hline $1(\mathrm{G})$ & IN & E, F, L & 320 \\
\hline $2(\mathrm{~K})$ & OUT & F, L & 453 \\
\hline $3(\mathrm{I})$ & OUT & F, L & 452 \\
\hline
\end{tabular}

FIgURE 5: An example of the initial PIT settings in the active state.

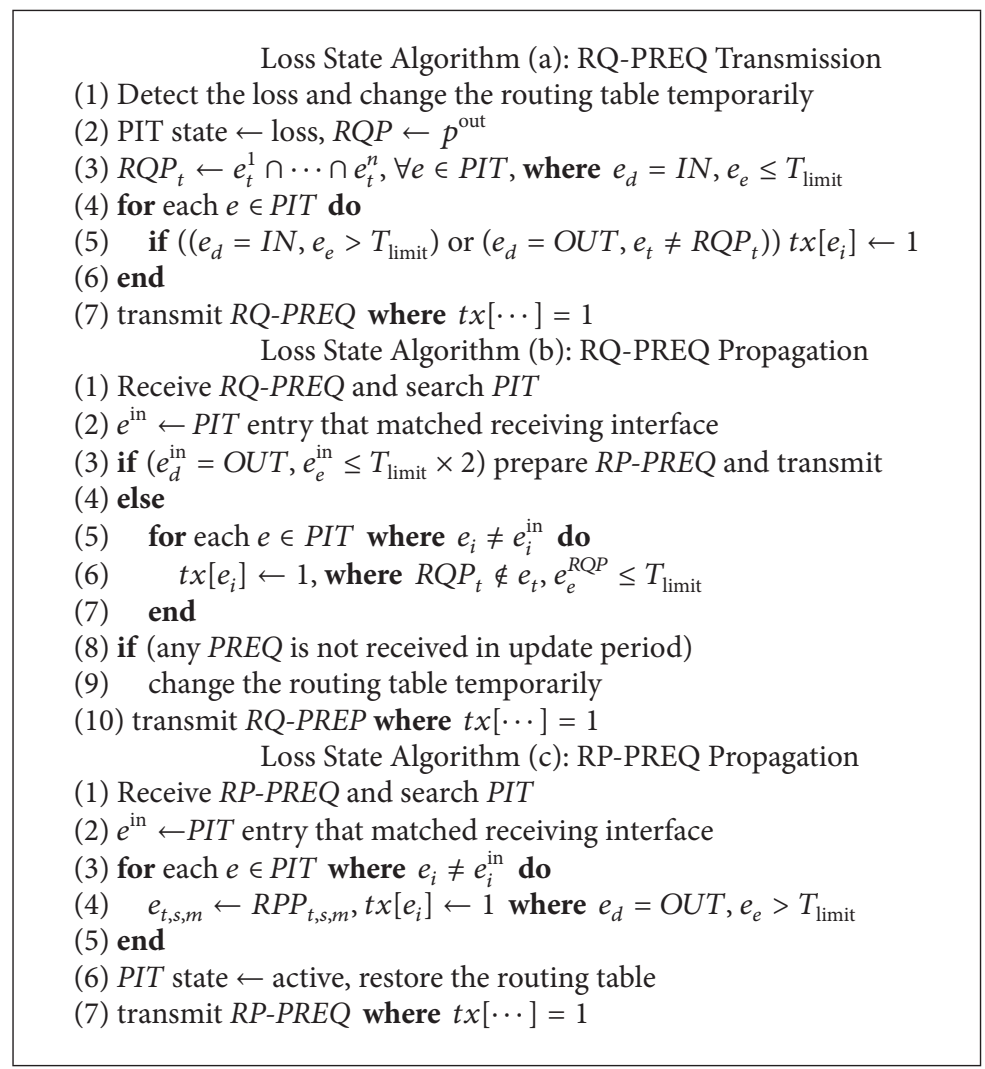

Algorithm 2: The algorithms for a PIT in the loss state.

has a loss state so the PIT can respond appropriately to that problem.

The proposed IA-AODV routing protocol uses the MGMT frame as the RQ-PREQ and the RP-PREQ to resolve the PREQ loss. The RQ-PREQ and RP-PREQ contain the path information, just like a general PREQ; the path modification and the recovery request and response can be conducted at the same time using these MGMT frames. The algorithm using the RQ-PREQ and RP-PREQ is presented in Algorithm 2.

First, the condition needed to transit from the active state to the loss state is that the PREQ is not received in the specific entry of the PIT during an update period. Algorithm 2(a) indicates the process of RQ-PREQ occurrence. In lines 1 to 3 


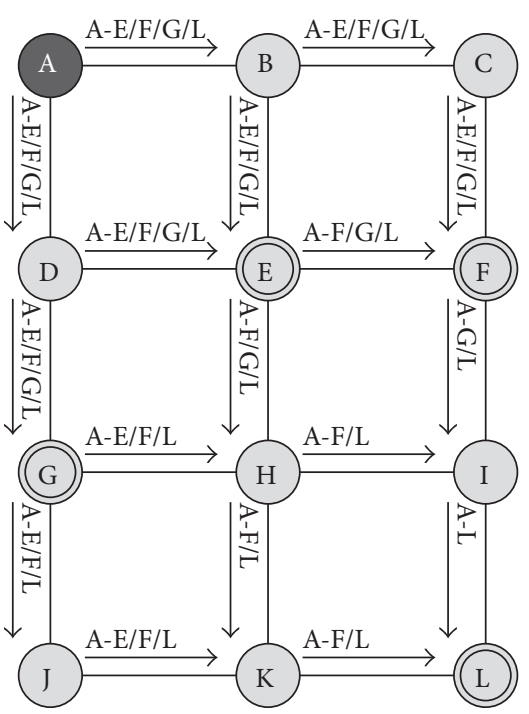

(a) PREQ propagation with the original PREQ prediction

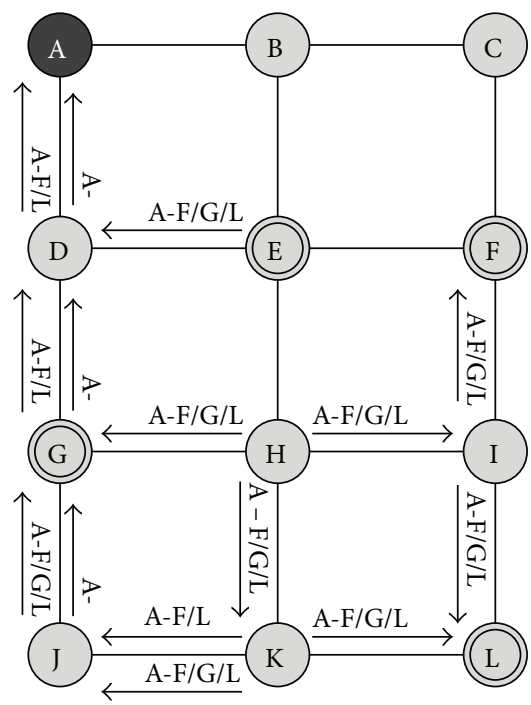

(c) RQ-PREQ is created in D, E, G, H, J, and K and then propagated

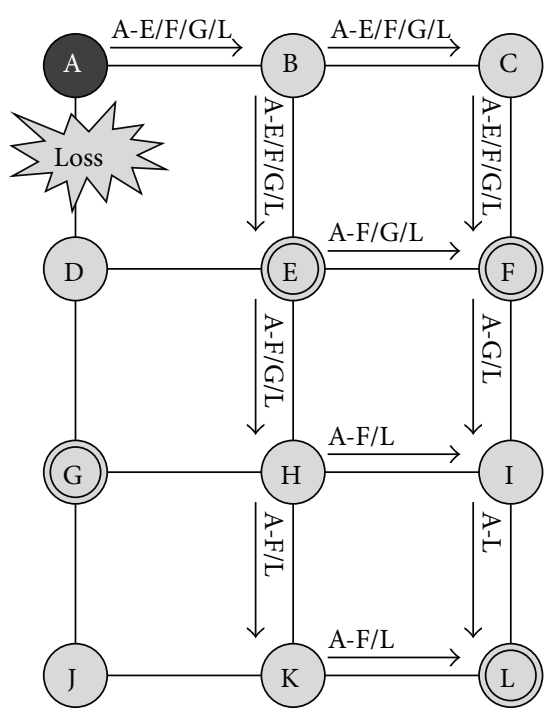

(b) Loss occurs in link A-D

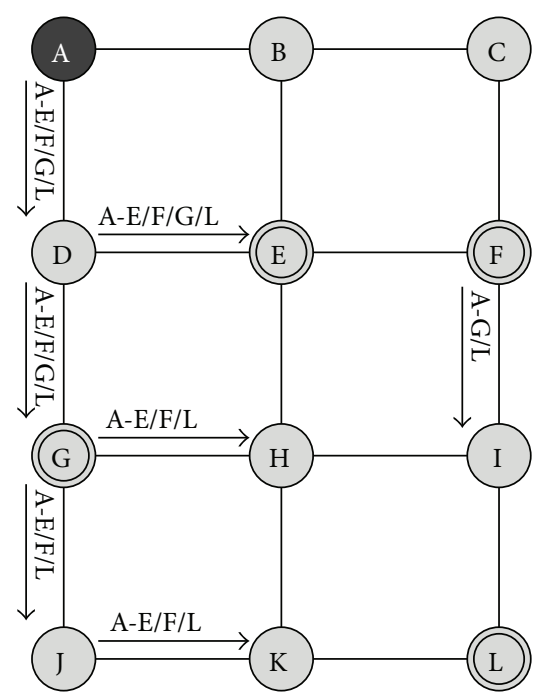

(d) RP-PREQ is sent by A, F, and recovery from loss state

FIGURE 6: An example of the PREQ loss recovery scheme.

of Algorithm 2(a), the mesh routing table will be temporarily changed if the loss is detected. Then, the RQ-PREQ is generated by using the PREQ that was recently sent, and then the target list of the RQ-PREQ is composed of the intersection of the target list of all PREQs that have been received normally. In lines 4 to 7, RQ-PREQ is sent through the IN interface that has not received a PREQ within the time limit and the OUT interface that has a different target list to the RQ-PREQ.

Algorithm 2(b) shows the process of RQ-PREQ propagation. In line 3 , if the role of the interface receiving the RQ-PREQ is OUT and the elapsed time of interface is not exceeding two times of the time limit, it means that the PREQ loss occurs in the link that comprises the corresponding interface. Therefore, the mesh node generates RP-PREQ and replies immediately. In particular, the node that has not received any PREQ within one update period temporarily replaces the routing table based on the information of the RQPREQ.

Finally, Algorithm 2(c) shows the process of RP-PREQ propagation. In lines 3 to 5 , the RP-PREQ is propagated using the OUT interface that has not transmitted the PREQ within the time limit. When this process is done, the PIT state changes from the loss state to active state and the routing table is also restored to the state before the change. However, if the node cannot receive the RP-PREQ within a certain time then the temporary modifications of the routing table will be maintained.

Figure 6 introduces the process of the PREQ loss recovery scheme. Figure 6(a) shows PREQ propagation along with 
the PREQ prediction scheme, and Figure 6(b) shows the occurrence of a PREQ loss in link A-D. In this case, nodes $D, G$, and J cannot receive any PREQs, and the specific IN interfaces of nodes $\mathrm{E}, \mathrm{H}$, and $\mathrm{K}$ also are not able to receive PREQs. Therefore, these six nodes conduct propagation of the RQ-PREQ as in Figure 6(c). The PITs in nodes D, E, G, $\mathrm{H}$, J, and $\mathrm{K}$ will transition to the loss state and will change the routing table temporarily.

Figure 6(d) shows propagation of the RP-PREQ. Nodes A and $\mathrm{F}$ can reply with RP-PREQ because those nodes received the RQ-PREQ through an OUT interface that sent the PREQ recently. The mesh node that received this RP-PREQ and that has a loss state PIT updates its own PIT and changes the state of the PIT to active. Also, it modifies the routing table to the state before the change and transmits the RP-PREQ via the proper interfaces. If the RP-PREQ is not received within the time limit in the mesh node that has the PIT in a loss state, the state of the PIT will be changed to inactive and all data of the PIT will be flushed. In addition, the temporary modification of the mesh routing table is maintained.

4.3. PREQ Sender Assignment Scheme. In the above sections, the MT-PREQ, the PREQ prediction scheme, and the PREQ loss recovery scheme are proposed in order to improve routing efficiency and network reliability in MIMC WMNs. In this section, we describe the scheme that reduces $N_{\text {src }}$ in (4) when the network application that generates the many internal network paths is serviced in the MIMC WMNs. The general path discovery process of the AODV is that the node that has data sends the PREQ to the target node, and the target node replies with PREP. The initial decision about the PREQ/PREP nodes is kept until that path is not used anymore. However, if many internal network paths are generated, all nodes will handle the process of sending only one PREQ and only one PREP in the worst case scenario. Then, $N_{\text {PREQ }}$ in (6) will increase up to the number of nodes in the network, which also increases routing complexity owing to the increase in the number of transfers required for routing. In addition, it may minimize the advantages of using MT-PREQ. Therefore, in this paper, a PREQ sender assignment scheme is proposed in order to resolve these issues.

First of all, the node that sends the PREQ frame is called the PREQ sender, and the node that replies with the PREP frame is called the PREP sender. Each node keeps the number of targets (TNUM) of the PREQ or PREP. In Figure 2, the TNUM of node $\mathrm{A}$ is 4 , and that of node $\mathrm{J}$ is 3 . When the new active path is required or the existing active path is disabled, the node sends the TNUM management frame, which contains the number of its own targets to all target nodes. The node receiving the TNUM frame from the target has to compare the number of its own targets. If the number of its own targets is bigger than the received TNUM, it will be a PREQ sender; otherwise, it becomes a PREP sender which waits for the PREQ. If the number of its own targets equals the received TNUM, it will be a PREQ or PREP sender by comparing the MAC address of the network interface card, which is a unique identifier.

Figure 7 shows the exchange of MGMT frames in the path discovery. Node A wants to send data to node B, so node A

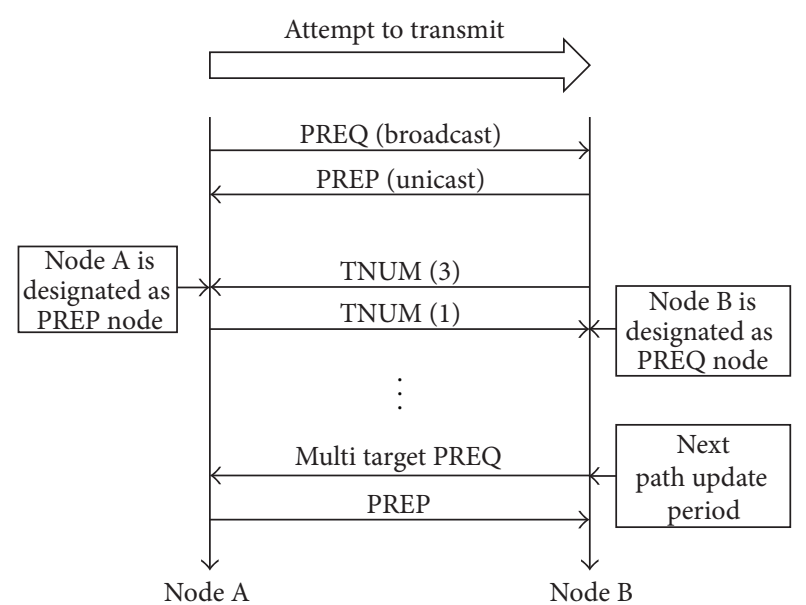

FIGURE 7: The operations by the PREQ sender assignment scheme.

transmits the PREQ, and B replies with PREP. After the path between $A$ and $B$ is established, nodes $A$ and $B$ exchange the TNUM frame containing the number of their own targets. In this process, node $A$ will be a PREP sender and node $B$ will be a PREQ sender. Then, node $B$ will transmit the MT-PREQ in every path update period; and node A replies with PREP for the path update.

For this scheme, each node keeps the number of its own active paths. If the number of active paths changes, then a TNUM frame will be transferred after the exchange of PREQ/PREP in the next path update. In order to exchange the number of active paths, the node that receives the TNUM replies as the TNUM frame. If the node changes from PREP sender to PREQ sender, the state of the PIT relating to the corresponding source node will have to be changed to inactive. By this, the IA-AODV can process routing using the minimum number of PREQs, regardless of centralized traffic or distributed traffic. Thus, it achieves improvement of routing efficiency.

\section{Experimental Results with a Real-World Test-Bed}

5.1. Implementation of the MIMC WMNs Using IA-AODV Routing Protocol. In this section, the implementation of a real-world test-bed and the experiment scenarios are described, and the results of experiments are analyzed to evaluate the IA-AODV routing protocol in MIMC WMNs. For implementation of the mesh router in MIMC WMNs, Ubiquiti's Routerstation Pro and Mikrotik's R52Hn were utilized as the network board and the network card, respectively. Routerstation Pro is a high-performance embedded board with the Atheros AR7161 $680 \mathrm{MHz}$ chipset; it is suitable for the target platform of the MIMC mesh router because it has three mini-PCI slots. R52Hn uses the AR9220 chipset and supports up to $300 \mathrm{Mbps}$ data rates at the PHY layer. In addition, the OpenWrt KAMIKAZE r22190 (Linux kernel 2.6.32.14) package was used for embedded Linux. The mesh engine was implemented by modifying compat-wireless-2.6.38-rc72 , which is a driver package containing ath9k and mac80211. 


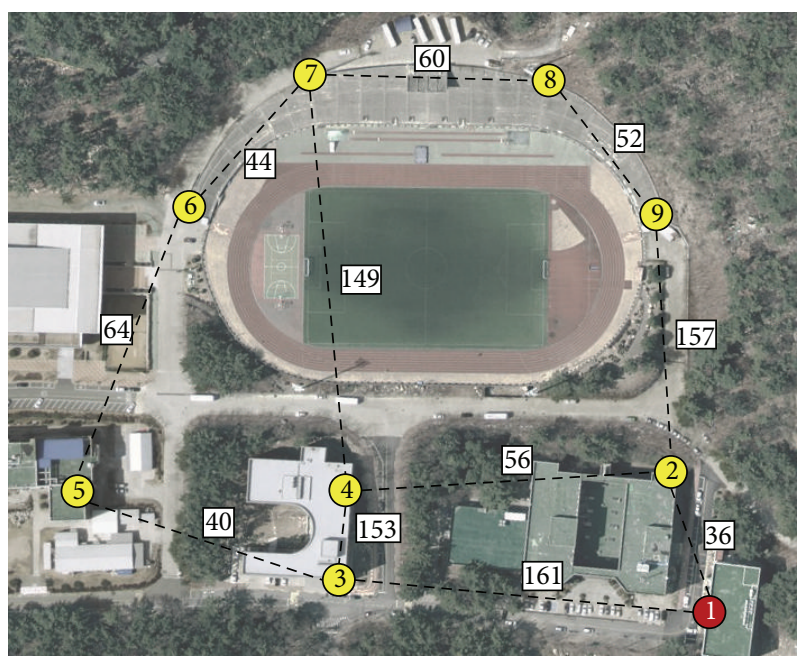

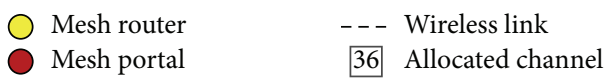

FIgURE 8: The topology of the outdoor test-bed for MIMC WMNs.

All experiments were performed on an outdoor testbed located at Pusan National University. The test-bed was constructed on the roof of each building and the playground, with mesh routers using IA-AODV. The test-bed was composed of nine mesh routers as seen in Figure 8, and each mesh router had the same number of interfaces as links. Each interface runs on the $5 \mathrm{GHz}$ channel and uses directional antennas. The topology is designed so that multiple multipaths can be generated.

5.2. Experiment Scenarios. The experiments used two scenarios (distributed traffic and centralized traffic) and were conducted to evaluate the efficiency of IA-AODV and the reliability of the MIMC WMNs. The number of MGMT frames, the ratio of routing malfunction, and the packet loss rate were measured in each experiment. All experiments used the iperf, which is a network performance measurement tool, and reported an average of the values that were performed 10 times; each experiment conducted UDP traffic transmission over two minutes. The source-destination pairs of UDP traffic were required to be distributed configuration for distributed traffic. In other words, nodes as many as possible were set as source or destination for the UDP traffic, so the traffic was configured to minimize the number of active paths in each node. In contrast, for the experiment using centralized traffic, the source-destination pairs were composed to be concentrated in a single node. Therefore, the traffic was organized that the active path was concentrated in a specific node.

\subsection{The Evaluation and Analysis of Experimental Results}

5.3.1. Experiments on Distributed Traffic. The topology in Figure 8 consists of 9 mesh nodes and 11 mesh links, and the experiment in this section makes the traffic maximize the distribution. Each mesh path becomes the active path if

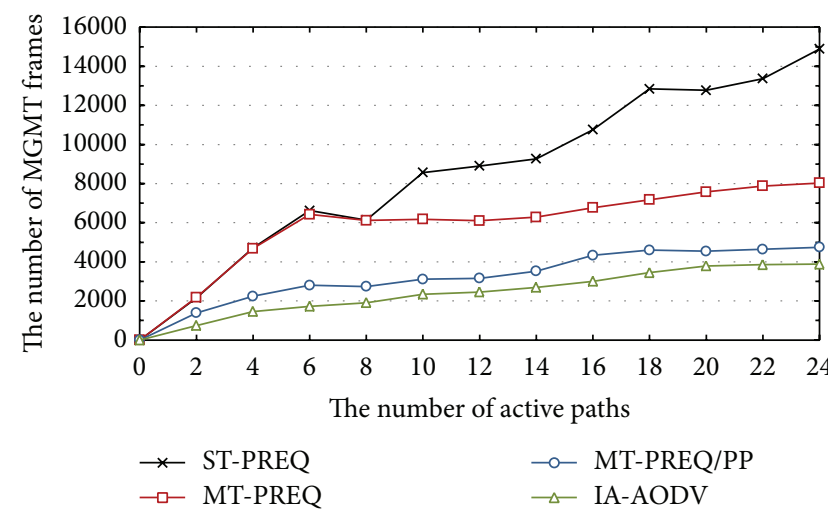

FIGURE 9: The number of MGMT frames needed for the path update process.

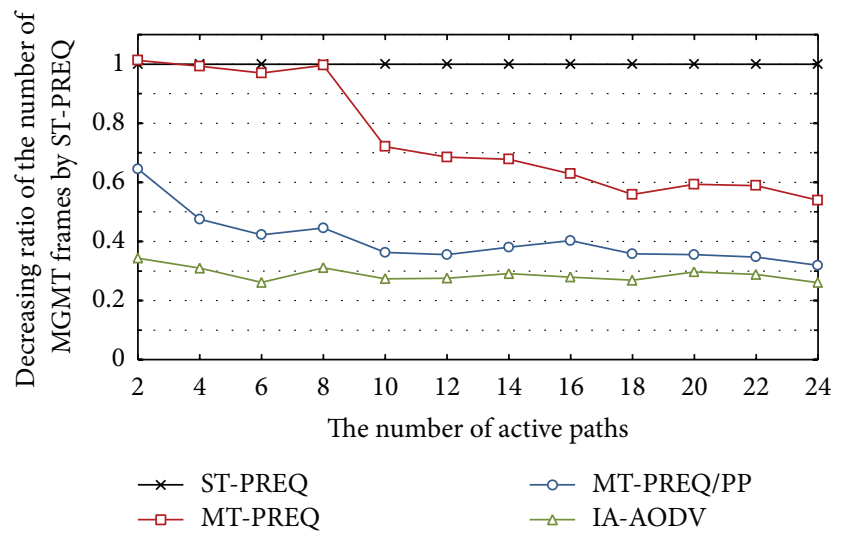

FIGURE 10: The decreasing ratio of the number of MGMT frames by ST-PREQ.

it is being used in communication, and it becomes inactive when communication is terminated. The experiment was set up to gradually increase the number of active paths. In the following experiments, ST-PREQ means the existing AODV routing protocol using ST-PREQ, and MT-PREQ signifies the existing AODV using MT-PREQ. Also, MT-PREQ/PP indicates the routing protocol used MT-PREQ and the PREQ prediction scheme. The IA-AODV proposed in this paper means the routing protocol that adopts full features, such as the MT-PREQ, the PREQ prediction scheme, the PREQ loss recovery scheme, and the PREQ sender assignment scheme. In addition, the number of MGMT frames of ST-PREQ, MTPREQ, and MT-PREQ/PP means the sum of transfers of PREQ/PREP in each node. However, the number of MGMT frames of IA-AODV is the sum of transmissions of PREQ, PREP, TNUM, RQ-PREQ, and RP-PREQ.

Figure 9 shows the number of MGMT frames in two minutes in the distributed traffic. Figure 10 presents the percentage of MGMT frames of each scheme compared to ST-PREQ. Each node in the test-bed, which is used for communication, has at least two active paths in case of two to eight total active paths in the distributed traffic scenario. Therefore, each node is responsible for sending PREQ and 


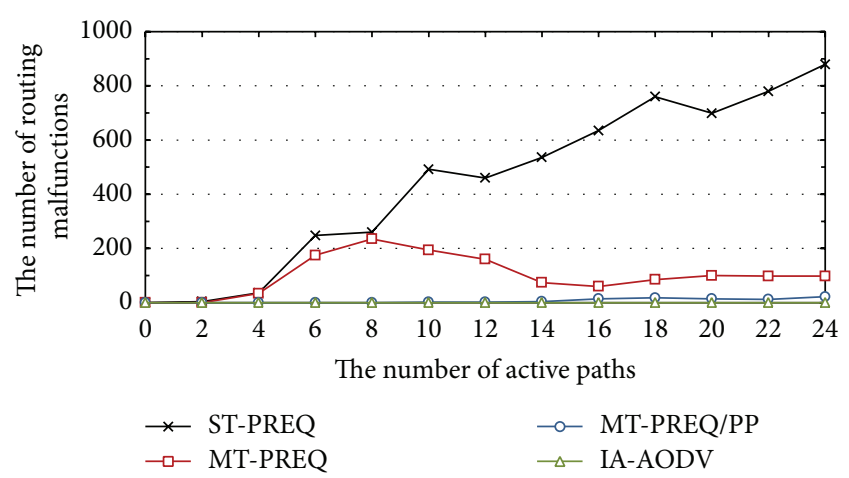

FIgURE 11: The number of routing malfunctions in 2 minutes.

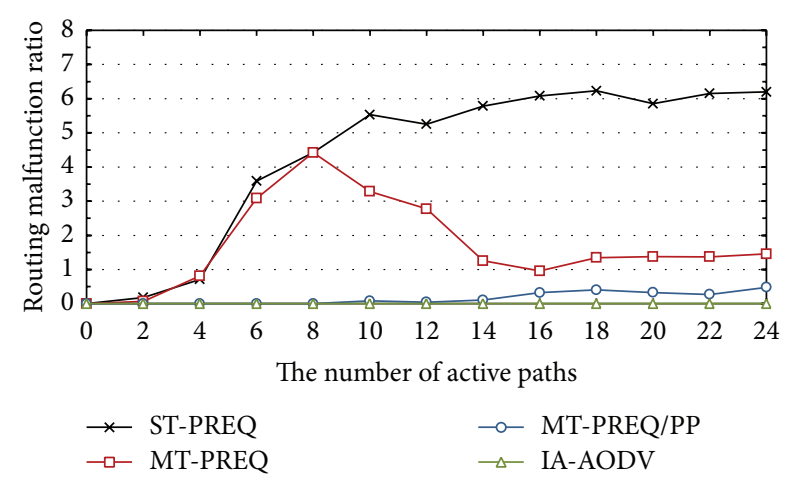

FIGURE 12: The routing malfunction ratio over distributed traffic.

PREP, so there are no differences between the number of MGMT frames using ST-PREQ and MT-PREQ in case of 2 to 8 total active paths. In terms of the number of PREQ targets, each node sends PREQs to only one target, so the MT-PREQ has no effect. However, the number of MGMT frames using the MT-PREQ is significantly reduced, when the effect of MTPREQ occurs. Since then, even if the number of active paths increases, the MT-PREQ can maintain routing using about $74.72 \%$ of the MGMT frames' transmission compared to the ST-PREQ.

In contrast, the routing protocol using the PREQ prediction scheme can manage routing using $40.57 \%$ of the MGMT frames, and the IA-AODV routing protocol compared to STPREQ is able to decrease the number of MGMT frames to update the path by $28.83 \%$. In particular, with IA-AODV, the TNUM frame, RQ-PREQ frame, and RP-PREQ frame are generated every time the active path changed. Therefore, if the active paths stay out longer, the number of MGMT frames used to perform the path update using IA-AODV can be reduced even more.

Figure 11 reports the number of routing malfunctions in 2 minutes, and Figure 12 shows the routing malfunction ratio. The routing malfunction as described in Section 3.2 means the mesh path changes to a poor path from loss of PREQ or the random receiving order of the PREQ, although the original path has no problem. The number of routing malfunctions means the sum of errors that occur in each

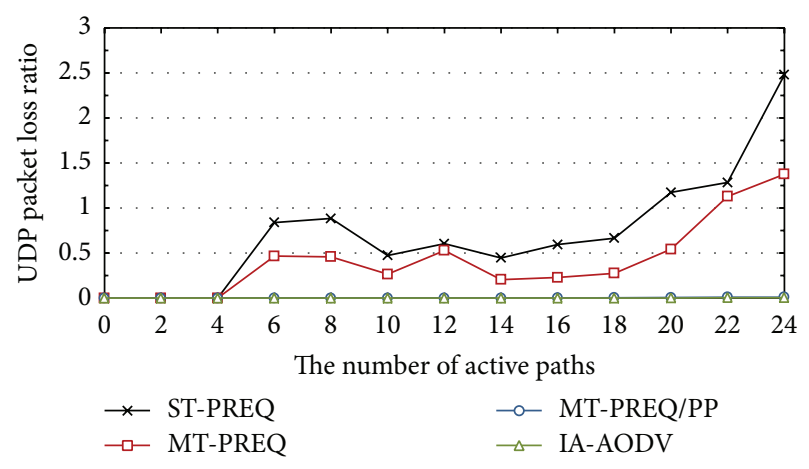

FIGURE 13: The packet loss ratio for distributed traffic.

link, and the routing malfunction ratio is the number of malfunctions over the number of PREQs and PREPs received, which can also be defined as

$$
\begin{aligned}
& \text { Routing Malfunction Ratio } \\
& =\frac{\text { The number of routing malfunction }}{\text { Received PREQ + Received PREP }} .
\end{aligned}
$$

Similar to the number of MGMT frames, the number of routing malfunctions and the routing malfunction ratio of ST-PREQ and MT-PREQ have similar results in 2 to 8 active paths, but after that, the difference increases. The average routing malfunction ratio is $4.67 \%$ using ST-PREQ and $1.85 \%$ using MT-PREQ. The reason for the higher ratio of routing malfunctions is that a structural problem exists when STPREQ is applied. Although the structural problem of AODV in MIMC WMNs is removed using MT-PREQ, the routing malfunction ratio of MT-PREQ is still relatively high due to the issue of PREQ random receiving order. When MT$\mathrm{PREQ} / \mathrm{PP}$ is used, the ratio is $0.17 \%$ on average. The ratio decreases because the interface assignment of the PREQ prediction scheme can nearly remove the probability that the PREQ random receiving order problem will occur. However, it cannot handle loss of PREQs, so network reliability is not perfectly guaranteed. The IA-AODV shows a $0 \%$ routing malfunction ratio because the loss of PREQs can be resolved with the PREQ loss recovery scheme.

Figure 13 shows that the UDP packet loss ratio follows the various numbers of active paths. Overall, the pattern looks similar to the graph of the routing malfunction rate, and the average loss rate is $0.787 \%$ using ST-PREQ, $0.457 \%$ using MTPREQ, $0.003 \%$ using MT-PREQ/PP, and $0.001 \%$ when IAAODV is used. When the IA-AODV is adopted in MIMC WMNs, the packet loss exists due to the network congestion from increasing traffic.

In other words, when the IA-AODV proposed in this paper is used in MIMC WMNs that have distributed traffic, compared to ST-PREQ, it can use $28.83 \%$ of the MGMT frames for the path update, perfectly removing the routing malfunction, and decreasing the average loss rate by $0.0013 \%$. Therefore, the proposed routing protocol can significantly improve not only routing efficiency but also network reliability. 


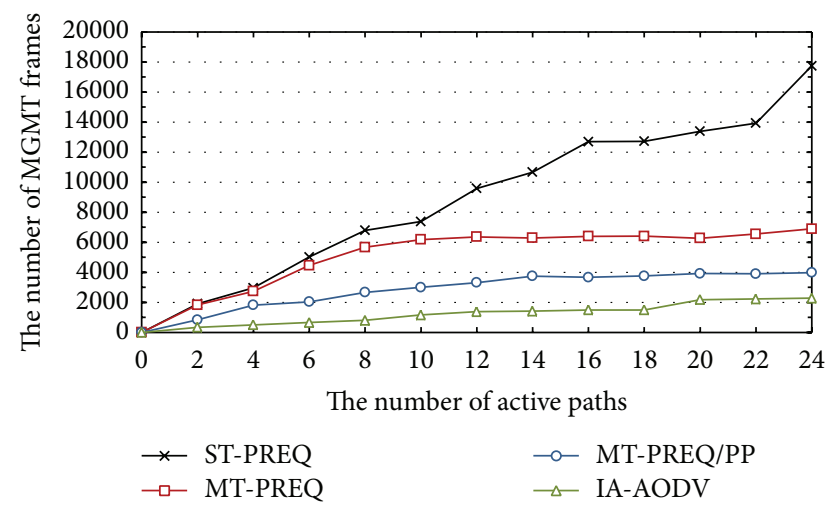

Figure 14: The number of MGMT frames for path update with centralized traffic.

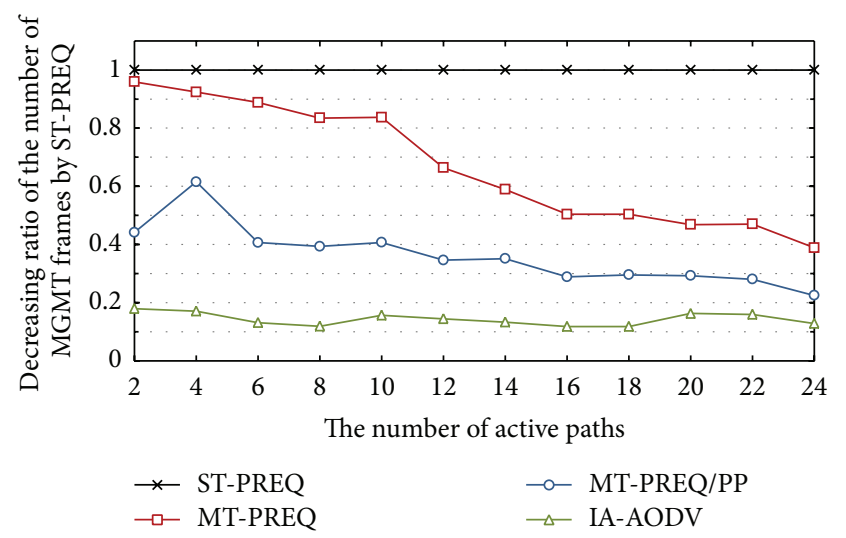

FIGURE 15: The decreasing ratio of the number of MGMT frames with the existing AODV.

5.3.2. Experiments on Centralized Traffic. For this scenario, the experiment was conducted with concentrated traffic, for this traffic form, UDP source, or destination assigned to a single node if possible. The experiment was performed with a gradual increase in the number of active paths.

Figure 14 shows the number of MGMT frames during two minutes from centralized traffics; Figure 15 shows the ratio of MGMT frames of each scheme compared to STPREQ. Though the UDP traffic is concentrated as much as possible, PREQ is sent by multiple nodes due to configuration so that the PREQ sender does not become one node. Thus, the efficiency of IA-AODV greatly increased compared to other routing protocols. Compared to ST-PREQ, the path update process utilized $66.91 \%$ of MGMT frames with MT-PREQ, $36.18 \%$ with MT-PREQ/PP, and $14.33 \%$ with IA-AODV. In particular, the efficiency of the PREQ sender assignment scheme will increase with centralized traffic.

When centralized traffic occurs, Figure 16 shows the number of routing malfunctions in two minutes, and Figure 17 shows the routing malfunction ratio. When using ST-PREQ the average routing malfunction ratio is $2.39 \%$, $1.22 \%$ using MT-PREQ, $0.40 \%$ using MT-PREQ/PP, and $0 \%$ using IA-AODV, like the experiments over distributed traffic. When the experiments over centralized traffic are

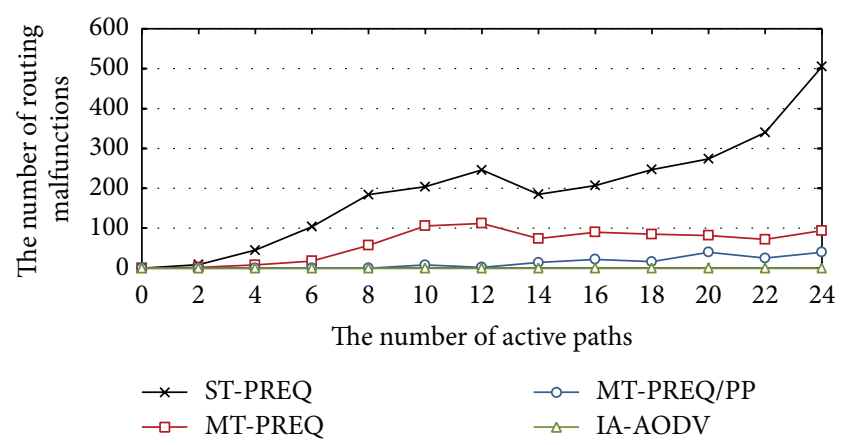

FIgURE 16: The number of routing malfunctions in 2 minutes over centralized traffic.

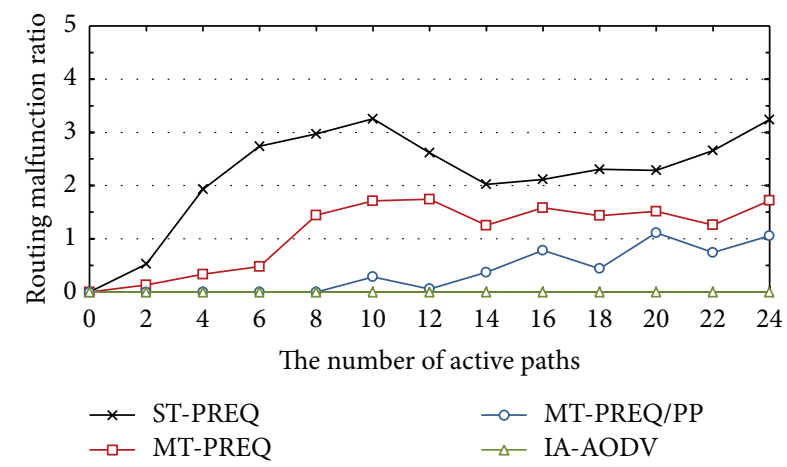

FIGURE 17: The routing malfunction ratio over centralized traffic.

compared to distributed traffic, the routing malfunction ratio was reported at a low level in every scheme.

Figure 18 shows that the UDP packet loss ratio follows the various numbers of active paths, which are formed with centralized traffic. Similar to distributed traffic, the result pattern looks like the routing malfunction ratio, and the average loss rate is $0.801 \%$ using ST-PREQ, $0.352 \%$ using MTPREQ, 0.150\% using MT-PREQ/PP, and 0.001\% using the IAAODV routing protocol. Also, the loss rate exists when using IA-AODV because of network congestion.

When the IA-AODV is adopted as a routing protocol in MIMC WMNs that have centralized traffic, similar to the experiment results over distributed traffic, it can update the path using only $14.33 \%$ of the MGMT frames, completely remove the routing malfunction, and decrease average loss rate by $0.0012 \%$.

\section{Conclusion}

In this paper, we define some issues that occur when the existing AODV routing protocol has been applied to a MIMC WMN environment, and we propose IA-AODV to resolve the above problems, thereby improving routing efficiency and network reliability. When the existing AODV is adopted in MIMC WMNs, problems occur, such as the structural problem, the random receiving order of PREQs, and the PREQ loss. The IA-AODV routing protocol based on interface assignment and MT-PREQ contains a PREQ 


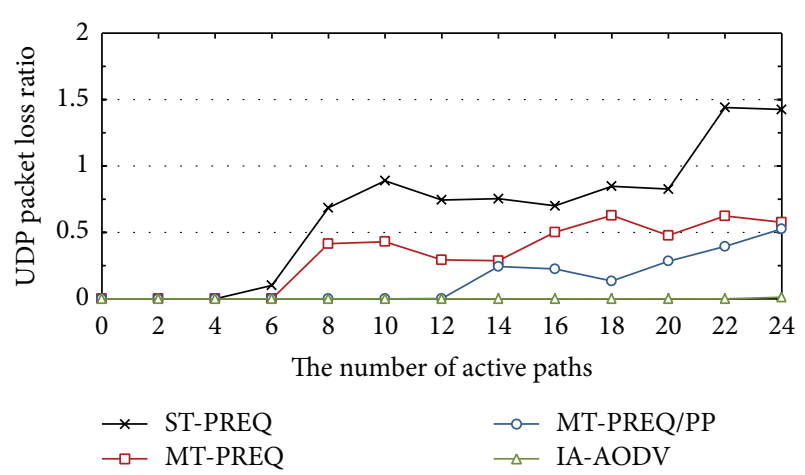

FIGURE 18: The packet loss ratio of each protocol over centralized traffic.

prediction scheme to assign the role of the interface, a PREQ loss recovery scheme to recover from PREQ loss, and a PREQ sender assignment scheme to alleviate routing complexity.

We implemented an outdoor test-bed for a MIMC WMN to evaluate IA-AODV and analyzed the proposed routing protocol from various angles. In an environment of distributed traffic, IA-AODV, compared to the existing AODV using ST-PREQ, is able to perform the path update with only $28.83 \%$ of the MGMT frames, perfectly remove the routing malfunction, and decrease average loss rate by $0.0013 \%$. Also, with centralized traffic, IA-AODV can conduct the path update using about $14.33 \%$ of the MGMT frames, completely eliminate the routing error, and decrease the loss rate by $0.0012 \%$. In other words, the proposed IA-AODV routing protocol has been able to significantly increase reliability and efficiency of MIMC WMNs.

One of the future directions we want to study is the effect caused by scaling up the network, such as scaling up the number of interfaces in each node and/or the number of nodes in the network. We will evaluate the computational cost for each node when the network size greatly increases.

\section{Expression by PREQ Prediction Algorithms}

PIT: $\quad$ PREQ information table

$e: \quad$ Entry of PIT

$e^{\text {in }}: \quad$ Entry which has IN direction

$P^{\text {in }}$ : Received PREQ

$P^{\text {out }}$ PREQ that will be sent

$x_{i, d, t, s, m, e}:$ The interface, direction, targets, $\mathrm{SN}$, metric, and exp_time of entry or PREQ, respectively

$m: \quad$ Metric

$m_{\text {last }}: \quad$ Metric of the last hop

$t: \quad$ Targets

$\operatorname{tx}[\cdots]: \quad$ The array that shows the interfaces used by transmission

RQP: RQ-PREQ

RPP: RP-PREQ

$T_{\text {limit }}: \quad$ The time limit used in loss decision.

\section{Conflict of Interests}

The authors declare that there is no conflict of interests regarding the publication of this paper.

\section{Acknowledgments}

This research was supported by Basic Science Research Program through the National Research Foundation of Korea (NRF) funded by the Ministry of Education, Science and Technology (2012R1A1A2043531). This research was supported by the MSIP (Ministry of Science, ICT and Future Planning), Korea, under the ITRC (Information Technology Research Center) Support Program (NIPA-2014-H0301-141048) supervised by the NIPA (National IT Industry Promotion Agency).

\section{References}

[1] IEEE, "Part 11: wireless LAN medium access control (MAC) and physical layer (PHY) specifications, amendment 10: mesh networking," IEEE 802.11s, IEEE Standards Association, 2011.

[2] A. Raniwala and T. Chiueh, "Architecture and algorithms for an IEEE 802.11-based multi-channel wireless mesh network," in Proceedings of the 24th Annual Joint Conference of the IEEE Computer and Communications Societies (INFOCOM '05), vol. 3, pp. 2223-2234, March 2005.

[3] A. Raniwala, K. Gopalan, and T. Chiueh, "Centralized channel assignment and routing algorithms for multi-channel wireless mesh networks," Mobile Computing and Communications Review, vol. 8, no. 2, pp. 50-65, 2004.

[4] P. Kyasanur and N. H. Vaidya, "Routing and interface assignment in multi-channel multi-interface wireless networks," in Proceedings of the IEEE Wireless Communications and Networking Conference (WCNC '05), pp. 2051-2056, IEEE, March 2005.

[5] P. Kyasanur and N. H. Vaidya, "Routing and link-layer protocols for multi-channel multi-interface ad hoc wireless networks," Mobile Computing and Communications Review, vol. 10, no. 1, pp. 31-43, 2006.

[6] G. Li, L. Yang, W. S. Conner, and B. Sadeghi, "Opportunities and challenges for mesh networks using directional antennas," in Proceedings of the 1st IEEE Workshop on Wireless Mesh Networks (WiMESH '05), IEEE, September 2005.

[7] U. Kumar, H. Gupta, and S. R. Das, "A topology control approach to using directional antennas in wireless mesh networks," in Proceedings of the IEEE International Conference on Communications (ICC '06), pp. 4083-4088, IEEE, Istanbul, Turkey, July 2006.

[8] C. E. Perkins and E. M. Royer, "Ad-hoc on-demand distance vector routing," in Proceedings of the 2nd IEEE Workshop on Mobile Computing Systems and Applications (WMCSA '99), pp. 90-100, IEEE, February 1999.

[9] B. Yu and H. Fei, "Performance impact of wireless mesh networks with mining traffic patterns," in Proceedings of the 5th International Conference on Fuzzy Systems and Knowledge Discovery (FSKD '08), pp. 493-497, IEEE, October 2008.

[10] S. Waharte, R. Boutaba, Y. Iraqi, and B. Ishibashi, "Routing protocols in wireless mesh networks: challenges and design considerations," Multimedia Tools and Applications, vol. 29, no. 3, pp. 285-303, 2006. 
[11] J.-S. Kim, S.-H. Chung, C.-W. Ahn, and W.-S. Kim, "Implementing channel-load aware routing scheme for IEEE 802.11 mesh networks," in Proceedings of the IEEE 7th International Conference on Wireless and Mobile Computing, Networking and Communications (WiMob '2011), pp. 525-528, October 2011.

[12] J.-S. Kim, S.-H. Chung, Y.-S. Lee, C.-W. Ahn, W.-S. Kim, and M.S. Jung, "Design and implementation of a WLAN mesh router based on multipath routing," in Proceedings of the International Conference on Information Networking (ICOIN '11), pp. 154-159, IEEE, Barcelona, Spain, January 2011.

[13] W. S. Kim and S. H. Chung, "Design and implementation of IEEE 802.11n in multi-hop over wireless mesh networks with multi-channel multi-interface," in Proceedings of the IEEE 9th International Conference on High Performance Computing and Communication (HPCC '12), pp. 707-713, IEEE, Liverpool, UK, June 2012.

[14] W.-S. Kim and S.-H. Chung, "Design of optimized AODV routing protocol for multi-interface multi-channel wireless mesh networks," in Proceedings of the 27th IEEE International Conference on Advanced Information Networking and Applications (AINA '13), pp. 325-332, Barcelona, Spain, March 2013.

[15] A. A. Pirzada and M. Portmann, "High performance AODV routing protocol for hybrid wireless mesh networks," in Proceedings of the 4th Annual International Conference on Mobile and Ubiquitous Systems: Computing, Networking and Services (MobiQuitous '07), pp. 1-5, IEEE, August 2007.

[16] A. A. Pirzada, M. Portmann, and J. Indulska, "Performance analysis of multi-radio AODV in hybrid wireless mesh networks," Computer Communications, vol. 31, no. 5, pp. 885-895, 2008.

[17] A. Valera, W. K. G. Seah, and S. V. Rao, "Cooperative packet caching and shortest multipath routing in mobile ad hoc networks," in Proceedings of the IEEE Societies 22nd Annual Joint Conference of the IEEE Computer and Communications (INFOCOM '03), vol. 1, pp. 260-269, March-April 2003.

[18] I. Chakeres and E. Belding-Royer, "AODV routing protocol implementation design," in Proceedings of the 24th International Conference on Distributed Computing Systems Workshops (ICDCSW '04), pp. 698-703, IEEE, March 2004.

[19] M. Singh and S. G. Lee, "Decentralized hybrid wireless mesh protocol," in Proceedings of the 2nd International Conference on Interaction Sciences: Information Technology, Culture and Human (ICIS '09), pp. 824-829, ACM, November 2009.

[20] S. J. Lee and M. Gerla, "AODV-BR: backup routing in ad hoc networks," in Proceedings of the Wireless Communications and Networking Conference (WCNC '00), pp. 1311-1316, IEEE, September 2000.

[21] A. P. Subramanian, M. M. Buddhikot, and S. Miller, "Interference aware routing in multi-radio wireless mesh networks," in Proceedings of the 2nd IEEE Workshop on Wireless Mesh Networks (WiMESH '06), pp. 55-63, IEEE, September 2006.

[22] R. Draves, J. Padhye, and B. Zill, "Routing in multi-radio, multihop wireless mesh networks," in Proceedings of the 10th annual international conference on Mobile computing and networking (MobiCom '04), pp. 114-128, ACM, September 2004.

[23] R. D. C. Paschoalino and E. R. M. Madeira, "A scalable link quality routing protocol for multi-radio wireless mesh networks," in Proceedings of the 16th International Conference on Computer Communications and Networks (ICCCN '07), pp. 1053-1058, Honolulu, Hawaii, USA, August 2007.
[24] T. Liu and W. Liao, "Interference-aware QoS routing for multirate multi-radio multi-channel IEEE 802.11 wireless mesh networks," IEEE Transactions on Wireless Communications, vol. 8, no. 1, pp. 166-175, 2009.

[25] T. C. Tsai and S. T. Tsai, "A cross-layer routing design for multiinterface wireless mesh networks," EURASIP Journal on Wireless Communications and Networking, vol. 2009, Article ID 208524, 2009.

[26] T. Anker, D. Dolev, and B. Hod, "Cooperative and reliable packet-forwarding on top of AODV," in Proceedings of the 4th International Symposium on Modeling and Optimization in Mobile, Ad Hoc and Wireless Networks (WiOpt '06), pp. 1-10, IEEE, April 2006.

[27] J. Boice, J. J. Garcia-Luna-Aceves, and K. Obraczka, “Combining on-demand and opportunistic routing for intermittently connected networks," Ad Hoc Networks, vol. 7, no. 1, pp. 201-218, 2009. 

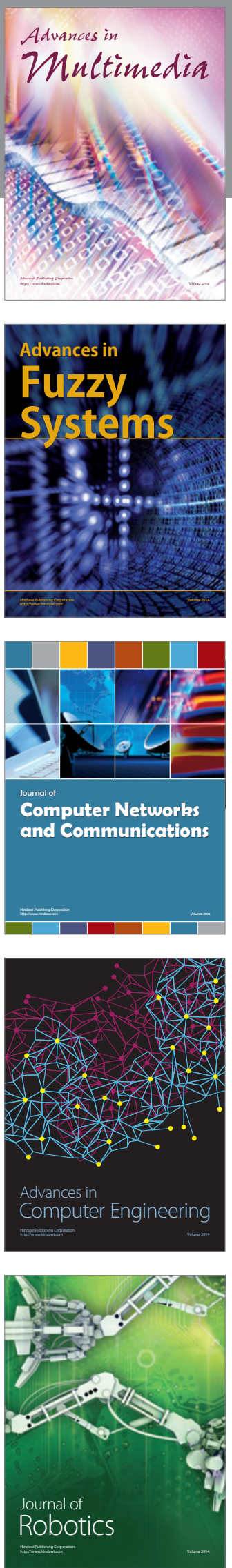

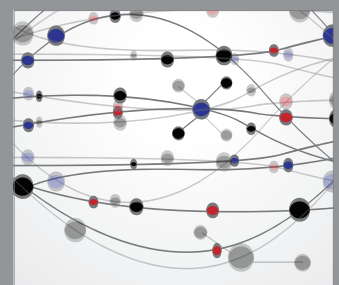

The Scientific World Journal
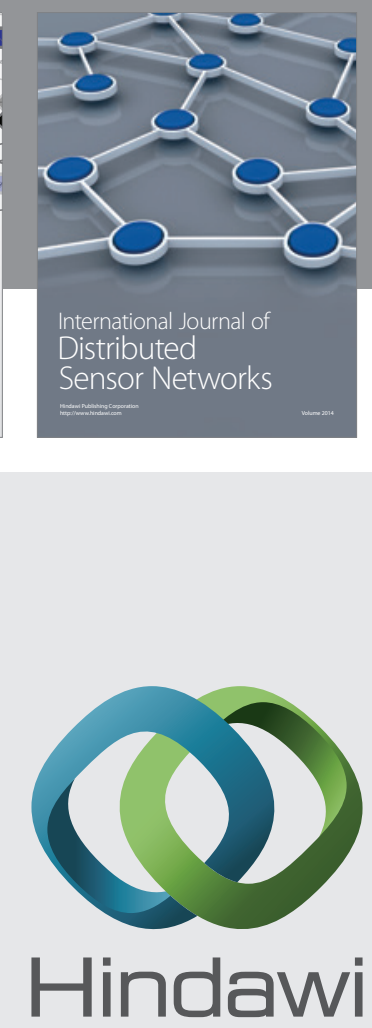

Submit your manuscripts at

http://www.hindawi.com
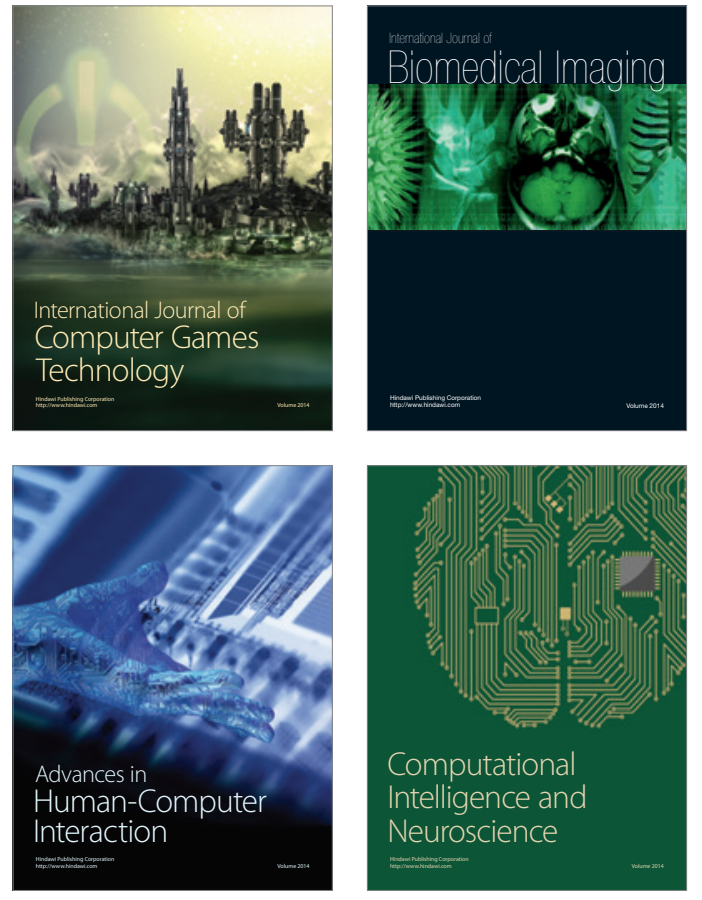
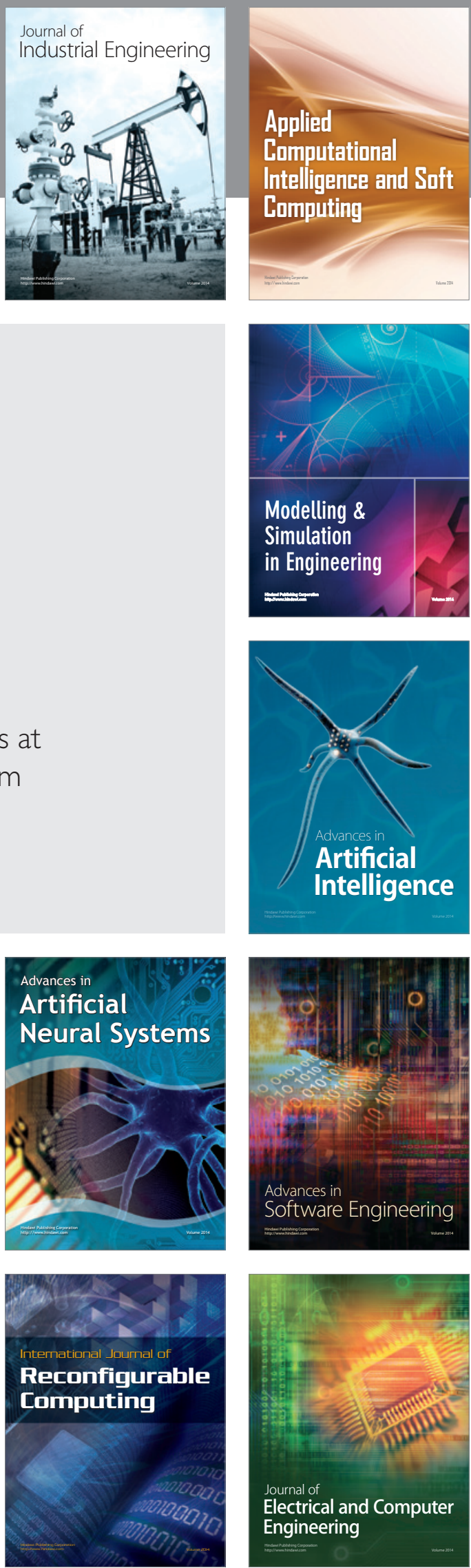